\title{
NEW FORM OF FAT EXTRACTION APPARATUS.
}

\section{By Bertram Blount.}

(Read at Meeting, May, 1888.)

Many kinds of fat extractors have been from time to time devised, some of which are specially suited for certain cases, but the form which has gained most favour (and deservedly so) for general purposes, is the Soxhlet.

This beautiful piece of apparatus is not, however, faultless, one imperfection being found in the fact that the liquid in contact with the substance to be extracted, particularly if it be one of the less volatile solvents, such as alcohol, is usually at a temperature considerably below its boiling point, to the detriment of its extractive powers.

Another weak point is the not infrequent irregularity in the action of the syphon, which will sometimes becorne filled as to its longer limb, with alternate portions of liquid and vapour, or air, and in consequenceempty the tube in which is the substance to be extracted, before the liquid in it has reached its proper level.

These failings led me to design the modification of it, which is here illustrated.

As may be seen in the cut, the essential parts of a Soxhlet, viz., a tube to contain the substance to be exhausted, and a syphon to empty it periodically, are retained.

These are, however, enclosed in an outer tube, which is fused to the inner tube at its upper end. The apparatus is attached by corks at its upper and lower ends to a vertical condenser and a flask, respectively, just like a Soxhlet.

The action is then as follows :-

The vapour of the liquid boiling in the flask passes up the annular space between the inner and outer tubes, and flows into the upper part of the inner tube through the holes AA (see cut). Thence it goes up into the condenser, and is there cooled, and falls back in drops into the inner tube, which is thus gradually filled; as the inner tube is completely jacketed with the vapour of the solvent, the liquid in it is kept at its boiling point the whole time it is in contact with the substance to be extracted. When the inner tube is filled to the level of the upper bend of the syphon it is, of course, emptied by it in the ordinary way, and the process of filling again begins.

I venture to think that this apparatus will be found to have several advantages over the ordinary Soxhlet, among which are the following :- 
(1) The solvent is kept at its boiling point in contact with the substance to be extracted.

(2) The syphon acts with great regularity; I am inclined to attribute this to its being kept at a uniform temperature throughout its length, and to the absence of any constriction, such as occurs in the case of the Soxhlet at the point where it passes through the wall of the lower tube connected with the flask.

(3) There is less liability of breakage, as it has no projecting parts, but presents a smooth cylindrical surface.

A good condenser with a wide tube should be used with it, as at the moment when the inner tube of the apparatus begins to empty itself, the liquid it contains (being just at its boiling point, and slightly agitated by the action of the syphon) partly vapourises, and thus throws additional work on the condenser.

It is to be noted that the rate at which drops fall from the condenser into the inner tube, does not measure the speed with which the latter is being filled, as a certain proportion of them do not originate from the condensation of the vapour sent up from the flask below, but from the liquid already in the inner tube.

The apparatus is made in two sizes, and can be obtained from Messrs. Townson and Mercer, 89, Bishopsgate Street, E.C., at the same price as an ordinary Soxhlet.

$\mathrm{AA}$ are two holes in the inner tube of the apparatus, affording means of communication with the outer tube.

$\mathbf{B}$ is a small projection from the inner tube, which, just touching the outer tube, "serves to keep the tubes concentric while the apparatus is being made, and afterwards to steady the inner tube.

$\mathbf{C}$ is the syphon, similar to that in an ordinary Soxhlet's apparatus. 